\title{
15-Hydroxyprostaglandin dehydrogenase is upregulated by hydroxychloroquine in rheumatoid arthritis fibroblast-like synoviocytes
}

\author{
HAK-JAE KIM ${ }^{1,2}$, SORA LEE ${ }^{3}$, HAW-YONG LEE ${ }^{1}$, HANSOL WON ${ }^{1}$, SUNG-HAE CHANG ${ }^{3}$ and SEONG-SU NAH $^{3}$ \\ ${ }^{1}$ Department of Clinical Pharmacology; ${ }^{2}$ Soonchunhyang Medical Research Institute; ${ }^{3}$ Division of Rheumatology, \\ Department of Internal Medicine, College of Medicine, Soonchunhyang University, \\ Cheonan, Choongcheongnam-do 330-930, Republic of Korea
}

Received August 8, 2014; Accepted May 13, 2015

DOI: $10.3892 / \mathrm{mmr} .2015 .3931$

\begin{abstract}
Hydroxyprostaglandin dehydrogenase (HPGD) is the key enzyme responsible for the metabolic inactivation of prostaglandin E2 $\left(\mathrm{PGE}_{2}\right)$ catabolism. $\mathrm{PGE}_{2}$ is one of the predominant catabolic factors involved in rheumatoid arthritis (RA). However, the expression and regulation of HPGD in RA fibroblast-like synoviocyte (FLS) remain to be elucidated. Disease-modifying anti-rheumatic drugs (DMARDs) are the most important anti-arthritic drugs, which reduce the effect of joint injury. The aim of the present study was to assess the expression of HPGD in RA tissues and cells, and normal synovial tissues and cells. The effect of the most popular DMARDs, hydroxychloroquine, on the expression of HPGD in RA-FLS was also investigated. Western blotting and immunohistochemical analysis demonstrated that the expression levels of HPGD in human synovium were lower in RA synovium compared with the normal and OA synovium. In RA-FLS, the expression of HPGD was increased following treatment with several DMARDs, including sulfasalazine, methotrexate, and hydroxychloroquine. Hydroxychloroquine $(10 \mu \mathrm{M})$ treatment induced the phosphorylation of ERK, SAPK/JNK and p38. Hydroxychloroquine induced a decrease in the release of $\mathrm{PGE}_{2}$, which was restored by mitogen-activated protein (MAP) kinase pathway inhibitors. Hydroxychloroquine may therefore, affect the pathogenesis of RA through the MAP kinase pathway by regulating the expression of HPGD.
\end{abstract}

Correspondence to: Professor Seong-Su Nah, Division of Rheumatology, Department of Internal Medicine, College of Medicine, Soonchunhyang University, 31 Suncheonhyang 6-gil, Dongnam-gu, Cheonan, Choongcheongnam-do 330-930, Republic of Korea

E-mail: so_ra2@nate.com

Key words: 15-hydroxyprostaglandin dehydrogenase, fibroblast-like synoviocyte, rheumatoid arthritis, hydroxychloroquine

\section{Introduction}

Rheumatoid arthritis (RA) is a chronic autoimmune disease characterized by inflammatory cell infiltration, synovial lining cell hyperplasia and hypertrophy, and a progressive destruction of cartilage and bone. Several patho-physiological mechanisms are involved in the development and progression of the disease (1). Rheumatoid joint exhibits a marked expression of synovial Prostaglandin $\mathrm{E}_{2}\left(\mathrm{PGE}_{2}\right)$ synthesizing enzymes, microsomal prostaglandin E2 synthase 1 (mPGES1), and cyclooxygenase $(\mathrm{COX}) 1$ and 2 (2). $\mathrm{PGE}_{2}$ is synthesized via multiple pathogenic mechanisms of RA. It acts as a mediator of pain and inflammation and promotes bone destruction (3). $\mathrm{PGE}_{2}$ synthesis is the result of the activities of COX and PGES. The biosynthesis and catabolism of prostaglandins is shown in Fig. 1 (4). COX1 and cytosolic PGES are constitutively expressed, whereas COX2 and mPGES1 are inducible in an inflammatory context (5). $\mathrm{PGE}_{2}$ is inactivated by the enzyme 15-hydroxyprostaglandindehydrogenase (HPGD) (6). HPGD is the key enzyme responsible for the biological inactivation of prostaglandins and associated eicosanoids. It has been reported that the downregulation of HPGD is associated with various types of cancer (7-10). HPGD synthesis was also found in mouse articular chondrocytes (11). However, the effect of this enzyme in the pathology of human RA remains to be elucidated.

Synthetic disease-modifying anti-rheumatic drugs (DMARDs) are a group of non-biological pharmacological agents, which can retard or inhibit the inflammatory disease process. This category comprises commonly used agents, including methotrexate (MTX), leflunomide, hydroxychloroquine (HCQ), sulfasalazine (SSZ) and gold salts (12). HCQ is a common disease-modifying therapeutic for RA (13) and may also be used safely in long-term treatments (14). However, the effect of hydroxychloroquine on $\mathrm{PGE}_{2}$ metabolism and the expression of HPGD remains to be elucidated.

The present study aimed to assess the expression levels of HPGD in RA tissues and fibroblast-like synoviocytes (FLS) compared with normal tissues and to determine whether the DMARDs regulated the expression of HPGD. The signal transduction pathway of activation by one of the DMARDs in RA-FLS was determined. 


\section{Materials and methods}

Primary cultures of human cells. Human cartilage samples were obtained from healthy individuals and patients with osteoarthritis (OA) or RA at the Soonchunhyang University Hospital (Cheonan, Korea). Primary culture was performed as previously described (15). Human articular cartilage was cut into small tissue slices and incubated and washed in medium prior to digestion with $0.1 \%$ collagenase (Invitrogen Life Technologies, Carlsbad, CA, USA) for $3 \mathrm{~h}$ at $37^{\circ} \mathrm{C}$. Following incubation, the slices of cartilage were almost completely digested. Undigested fragments were removed by passing the solution through a nylon mesh (70 $\mu \mathrm{m}$ nylon; BD Falcon, Bedford, MA, USA). The isolated cells were washed three times by centrifugation at $211 \mathrm{x}$ g for $10 \mathrm{~min}$ and were resuspended in phosphate buffered saline (PBS; $\mathrm{pH}$ 7.4). Following culturing for 4 days, the cells were starved in serum-free Dulbecco's modified Eagle's medium (DMEM) with D-glucose, L-glutamine, sodium pyruvate and sodium bicarbonate, supplemented with $20 \%$ fetal bovine serum (FBS), $100 \mathrm{U} / \mathrm{ml}$ penicillin and $100 \mu \mathrm{g} / \mathrm{ml}$ streptomycin, for $24 \mathrm{~h}$ at $37^{\circ} \mathrm{C}$. The morphological features and the expression levels of type II collagen and aggrecan were consistent with a chondrocyte phenotype, as opposed to fibroblast-like cells. The cells were passaged upon reaching confluence by gentle trypsinization and the cells were used for experiments between passage 4 and 8 .

Immunohistochemistry. Tissue samples obtained from human synovium were fixed in $10 \%$ paraformaldehyde in PBS and were prepared using the routine method with paraffin blocks (16). To perform immunohistochemical staining for HPGD, a $9 \mu \mathrm{m}$ paraffin block was deparaffined, remoisturized and placed in $0.01 \mathrm{M}$ citrate buffer solution, with subsequent heating using ultra short waves (Microwave Processor; cat. no. B35600001; Thermo Fisher Scientific, Erembodegem, Belgium) at $100^{\circ} \mathrm{C}$ for $20 \mathrm{~min}$. The tissue sections were incubated with a mixture of methanol and $0.3 \% \mathrm{H}_{2} \mathrm{O}_{2}$ in methanol in order to remove the intrinsic peroxidase activity. The tissue sections were subsequently immunohistochemically stained using an UltraTech kit (Immunotech, Marseille, France), according to the manufacturer's instructions. The tissue sections were pretreated with $1 \%$ bovine serum albumin in PBS and were incubated with the HPGD antibody (1:100; cat. no. 160615; Cayman Chemical Co., Ann Arbor, MI, USA). Following antibody incubation, the tissue sections were incubated with biotinylate secondary antibody (1:250; cat. no. sc-2042; Santa Cruz Biotechnology, Inc., Santa Cruz, CA, USA), washed with PBS and treated with peroxidase-conjugated streptavidin. The tissue sections were then incubated with 3,3'-diaminobenzidine tetrahydrochloride, containing $0.05 \% \mathrm{H}_{2} \mathrm{O}_{2}$ for $3 \mathrm{~min}$ and counterstained with hematoxylin. Image quantification was performed with Image J software (NIH, Bethesda, MD, USA), as previously described, with minor modifications (17). Briefly, the pixel intensities in the enlarged images (magnification, x200) were calibrated by setting the display value range from 0 (black) to 255 (red). The threshold level for detection was selected by viewing histograms and adjusted to distinguish the intensity of the signal from that of the non-specific background. An identical threshold level was applied to all the images to allow valid comparison of normal, OA and RA images. The intensity of the labeling was determined using 700-pixel boxes randomly placed at different locations in the labeled area. The background intensity was determined using boxes positioned in areas of no signal.

Immunocytochemistry. The RA-FLS were grown and subsequently incubated in a 24 -well tissue culture plate $\left(2 \times 10^{4}\right.$ cells/ well) for immunofluorescence experiments. The cell culture medium was removed once the cells had grown to $80 \%$ and the cells were serum-starved for $2 \mathrm{~h}$ and subsequently treated with DMARDs for $24 \mathrm{~h}$. The cells were washed twice with sterile PBS and were subsequently fixed with $4 \%$ paraformaldehyde in PBS for 30 min at room temperature, followed by washing twice with sterile PBS. The fixed cells were blocked in 5\% horse serum for $1 \mathrm{~h}$ at room temperature, followed by washing twice with sterile PBS. An antibody against HPGD (1:100; Cayman Chemical, Co.) in PBS was added to the cells and incubated overnight at $4^{\circ} \mathrm{C}$. The cells were washed three times with PBS and incubated with Cy3-conjugated anti-goat secondary antibody (1:500; Jackson Immuno Research Laboratories, Inc., West Grove, PA, USA) for $1 \mathrm{~h}$ at room temperature. The cells were mounted following washing. Fluorescence images were captured using a confocal laser scanning microscope (FV10-ASW; Olympus, Tokyo, Japan). Quantification was performed previously described, with minor modifications (17).

Briefly, the images (magnification, x200) were analyzed using Image J software (NIH). The pixel intensity was calibrated by setting the display value ranging from 0 (black) to 255 (red). The threshold level remained constant for all the images to allow comparison of images in normal, OA and RA-FLS samples. The pixel intensity of the labeling was determined by randomly positioning boxes (300 square pixels) around the labeling at different locations on the image.

Western blot analysis. The cells were cultured in a $10 \mathrm{~cm}$ culture dish to $\sim 80 \%$ confluence $\left(1 \times 10^{6}\right.$ cells/well $)$ and were starved in DMEM without FBS for $24 \mathrm{~h}$. The cells were subsequently incubated for $24 \mathrm{~h}$ in the presence of DMARDs. Wortmannin, PD98059 and SB203580 (A.G. Scientific, Inc., San Diego, CA, USA) were added to the RA-FLS $30 \mathrm{~min}$ prior to HCQ stimulation. Unstimulated cells were used as controls. Following stimulation with different compounds, the cells were harvested and lysed with lysis buffer, containing $1 \%$ sodium deoxycholate in $150 \mathrm{mM} \mathrm{NaCl}, 10 \mathrm{mM}$ Hepes (pH 7.4), $0.1 \%$ sodium dodecyl sulfate (SDS), $1 \%$ Triton X-100, $5 \mathrm{mM}$ ethylenediaminetetraacetic acid, $20 \mu \mathrm{g} / \mathrm{ml}$ aprotinin, $20 \mu \mathrm{g} / \mathrm{ml}$ leupetin and $1 \mathrm{mM}$ PMSF, on ice. The lysed protein was separated on 10-20\% SDS-polyacrylamide gel electrophoresis gels and transferred onto a polyvinylidene fluoride membrane. Following blocking with 5\% skim milk for $1 \mathrm{~h}$ at room temperature, the membranes were probed with antibodies against HPGD (1:200; Cayman Chemical Co.), COX2 (1:200; Cayman Chemical Co.), COX1 (1:500; Cayman Chemical Co.), mPGES1 (1:250; Cayman Chemical Co.), phospho-ERK, p38, SAPK/JNK (1:1,000; Cell Signaling Technology, Inc., Danvers, MA, USA) and $\beta$-actin (ACTB; 1:5,000; Santa Cruz Biotechnology, Inc., Santa Cruz, CA, USA) overnight at $4^{\circ} \mathrm{C}$. Following this, the membrane was washed in TBST, 
containing $50 \mathrm{mM}$ Tris- $\mathrm{HCl}(\mathrm{pH} 7.4), 150 \mathrm{mM} \mathrm{NaCl}$ and $0.1 \%$ Tween-20, and incubated with peroxidase-conjugated secondary antibody (1:2,000; Santa Cruz Biotechnology, Inc.) for $1 \mathrm{~h}$ at room temperature. The membranes were incubated with Western Bright enhanced chemilluminescence kit (Advansta, Inc., Menlo Park, CA, USA) and exposed to $\mathrm{X}$-ray films. The images were captured using a ChemiDoc Imaging system $\left(\right.$ ChemiDoc $^{\mathrm{TM}} \mathrm{XRS}+$ System with Image Lab $^{\mathrm{TM}}$ Software; Bio-Rad, Hercules, CA, USA). Quantitative measurements of the protein expression levels of HPGD and ACTB were performed using Image J software (NIH). The mean pixel intensities of HPGD and ACTB were measured by positioning a box around the protein band and subtracting the background intensity. The integrated density values were presented as the mean \pm standard deviation between individual protein levels normalized against the integrated density value of ACTB.

Cell viability assay. The cell viability of FLS was accessed using a 3-(4,5-dimethylthiazol-2-yl)-5-(3-carboxymethoxy phenyl)-2-(4-sulfophenyl)-2H-tetrazolium (MTS) assay kit (Promega, Madison, WI, USA), according to the manufacturer's instructions. The FLS $\left(1 \times 10^{5}\right.$ cells/100 $\mu 1 /$ well $)$ were grown in 96-well microtiter plates for $24 \mathrm{~h}$. HCQ was added directly to the culture media and the cells were treated with various concentrations of HCQ for $24 \mathrm{~h}$. The MTS cell proliferation assay reagent was added and the samples were incubated at $37^{\circ} \mathrm{C}$ in $5 \% \mathrm{CO}_{2}$ for $4 \mathrm{~h}$. The absorbance was measured at $490 \mathrm{~nm}$ using GloMax-Multi Microplate Multimode Reader (Promega), and the difference between the test and reference wavelength was calculated. The cell viability was calculated using the equation: (optical density ratio of HCQ-treated sample/non-treated sample) x $100(\%)$.

Enzyme immunoassay to measure $P G E_{2}$. The RA-FLS $\left(1 \times 10^{5}\right.$ cells) were grown in 24-well plates and were serum starved overnight prior to stimulation with HCQ. Following washing with PBS, the cells were pretreated with $\mathrm{HCQ}$ at $37^{\circ} \mathrm{C}$ for $24 \mathrm{~h}$ in DMEM in an atmosphere of $5 \% \mathrm{CO}_{2}$. The culture supernatant described above was collected at day 1 . The level of $\mathrm{PGE}_{2}$ in the medium was determined using a $\mathrm{PGE}_{2}$ parameter assay kit (R\&D systems, Minneapolis, MN, USA), according to the manufacturer's instructions.

Statistical analysis. The data are presented as the mean \pm standard deviation. Statistical analysis was performed using SPSS 13.0 software (SPSS Inc., Chicago, IL, USA). Groups were compared using the Student's t-test. $\mathrm{P}<0.05$ was considered to indicate a statistically significant difference.

\section{Results}

The expression levels of HPGD in normal, $O A$ and RA synovial tissues were analyzed by immunohistochemical staining. HPGD expression in normal, OA and RA synovial tissues was analyzed by immunohistochemical staining (Fig. 1A). These images were quantified using Image J software (Fig. 1B). Immunohistochemical staining revealed that the expression levels of HPGD among the normal synovial tissue staining were marked and extensive, whereas staining in RA synovial

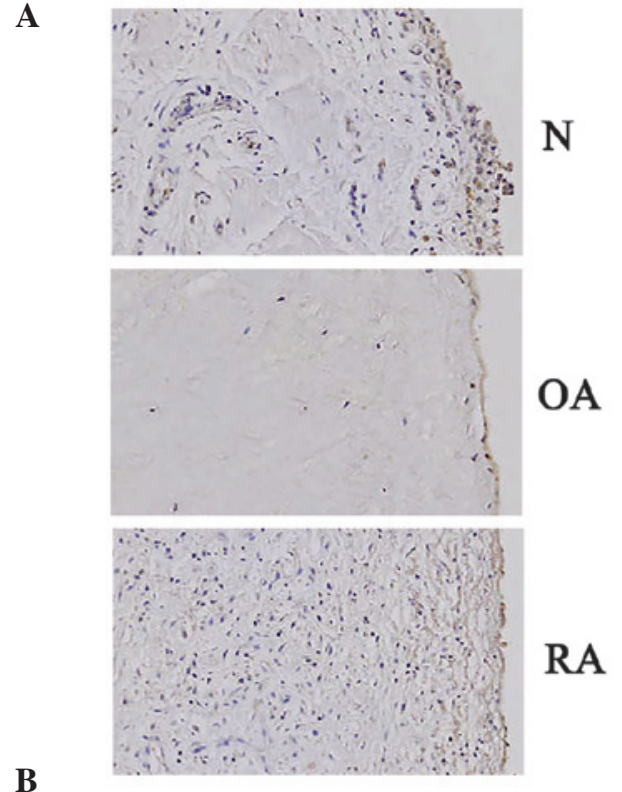

B

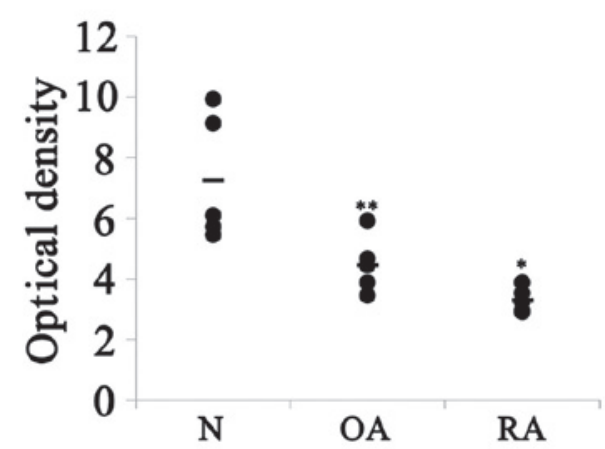

Figure 1. Expression of HPGD in the synovial tissue from $\mathrm{N}$ individuals, patients with OA and patients with RA. (A) Immunohistochemical staining revealed positive (brown) staining for HPGD in synovial tissue from $\mathrm{N}$ individuals, patients with OA and patients with RA (magnification, $x 200 ; n=5$ ). (B) Quantitation of the images from using ImageJ software ${ }^{*} \mathrm{P}<0.001$, ${ }^{* *} \mathrm{P}=0.009$, compared with $\mathrm{N}$ ). N, normal; OA, osteoarthritis; RA, rheumatoid arthritis; HPGD, 15-hydroxyprostaglandin dehydrogenase.

tissue was weak and confined predominantly to the synovial membrane. In addition, inflammatory infiltrates appeared in RA synovial tissue. Normal, OA and RA-FLS were isolated from human synovial tissues. The expression levels of HPGD expression were investigated by immunofluorescence in the FLS from each synovial tissue (Fig. 2A). The immunocytochemical results demonstrated that the antibodies of HPGD exhibited positive reactions in normal FLS, while little fluorescence signal was observed in RA-FLS (Fig. 2B; $\mathrm{P}<0.001$ ). The protein expression levels of HPGD in the normal, OA and RA-FLS tissues were confirmed by western blotting (Fig. 2C). Western blot analysis demonstrated that the protein expression levels of HPGD was lower in RA-FLS compared with normal and OA-FLS. An average of 1.8- and 1.3-fold less expression of HPGD was demonstrated in the RA-FLS (Fig. 2D).

Several DMARDs were assessed for their ability to induce the expression levels of HPGD in RA-FLS. The RA-FLS were cultured in a $100 \mathrm{~mm}$ dish up to $~ 80 \%$ confluence and were subsequently starved in DMEM without FBS for $2 \mathrm{~h}$. The cells were incubated for $24 \mathrm{~h}$ in SSZ $(10 \mu \mathrm{g} / \mathrm{ml})$, HCQ $(10 \mu \mathrm{M})$, MTX $(10 \mathrm{nM})$ and infliximab (10 ug/ml). Unstimulated cells 
A
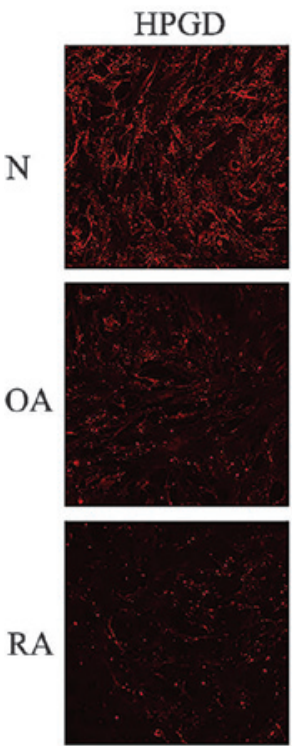

DAPI
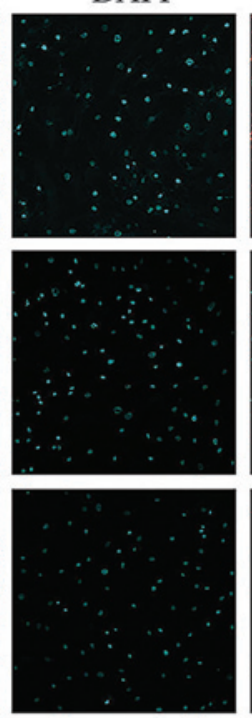
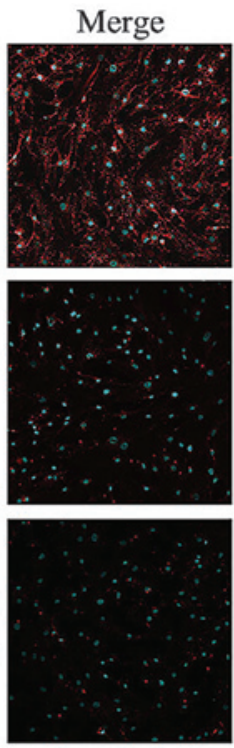

B

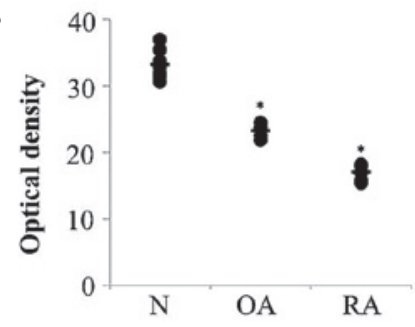

C

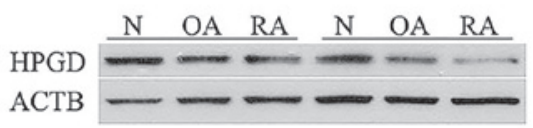

D

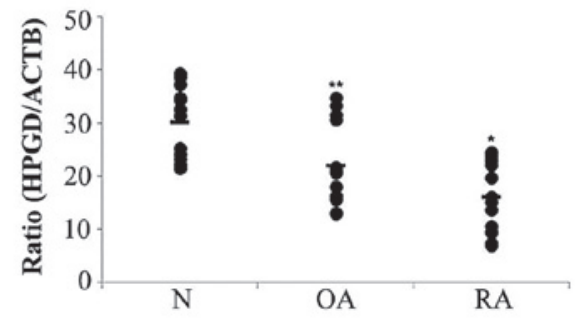

Figure 2. Expression of HPGD in FLS from N individuals, patients with OA and patients with RA. (A) Confocal microscopy images demonstrated immunohistochemical staining for HPGD (red; Cy3) with DAPI, in FLS (scale bar, $50 \mu \mathrm{m}$; n=12). (B) Quantification of the images from (A) using ImageJ software. (C) The expression of HPGD in the FLS from N individuals, patients with OA and patients with RA. ACTB was used as an internal control. (D) Graph demonstrating the ratio of HPGD to ACTB as determined by ImageJ software ("P $<0.001,{ }^{* *} \mathrm{P}=0.005$, compared with $\left.\mathrm{N}\right)$. N, normal; OA, osteoarthritis; RA, rheumatoid arthritis; HPGD, 15-hydroxyprostaglandin dehydrogenase; FLS, fibroblast-like synoviocytes, ACTB, $\beta$-actin.

A

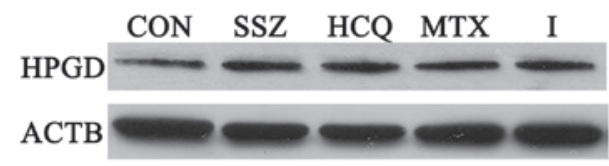

B

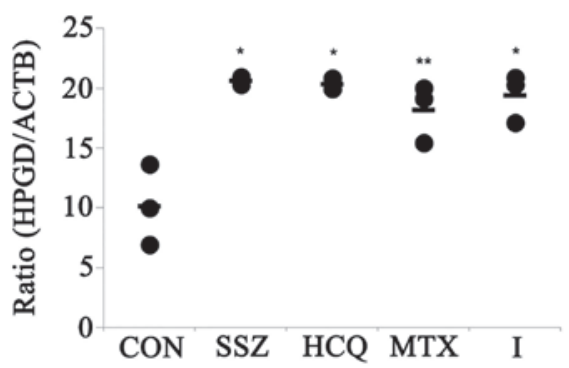

Figure 3. Effects of disease modifying antirheumatic drugs on the expression of HPGD in the RA-FLS. (A) The expression of HPGD in the RA-FLS was determined by western blotting $(n=4)$. RA-FLS were serum-starved for $2 \mathrm{~h}$ and subsequently treated with SSZ $(10 \mu \mathrm{g} / \mathrm{ml})$, HCQ $(10 \mu \mathrm{M})$, MTX (10 nM) or I $(10 \mu \mathrm{g} / \mathrm{ml})$ for $24 \mathrm{~h}$. (B) The graphs revealed the ratio of HPGD to ACTB as determined by ImageJ software $\left({ }^{*} \mathrm{P} \leq 0.001,{ }^{* *} \mathrm{P}=0.003\right.$, compared with control). CON, control; SSZ, sulfasalazin; HCQ, hydroxychloroquine; MTX, methotrexate; I, infliximab; HPGD, 15-hydroxyprostaglandin dehydrogenase; ACTB, $\beta$-actin; RA-FLS, rheumatoid arthritis fibroblast-like synoviocytes.

were used as controls. Western blot analysis demonstrated that all DMARDs increased the expression levels of HPGD > 2-fold compared with the untreated control cells $(\mathrm{P}<0.05$; Fig. 3$)$.

The cell viability in RA-FLS was assessed to obtain the appropriate concentration of HCQ for further experiments (Fig. 4). Cell viability was measured using an MTS assay kit, according to the manufacturer's instructions. The cells were treated with various concentrations of HCQ $(1,10,20$, and $50 \mu \mathrm{M})$. The results demonstrated that cell viability was decreased by treatment with HCQ in a dose-dependent manner (Fig. 4A-E). Cell viability was $89.3 \pm 7.4,87.7 \pm 1.6,85.8 \pm 1.1$ and $30.2 \pm 1.8 \%$ compared with the control at $1,10,20$ and $50 \mu \mathrm{M}$ HCQ, respectively (Fig. 4F).

$\mathrm{PGE}_{2}$ release following HCQ stimulation in the RA-FLS was measured using an enzyme immunoassay (Fig. 5). The cells were treated with various concentrations of HCQ $(1,10$, 20 and $50 \mu \mathrm{M})$ prior to harvesting of the supernatants. The quantities of $\mathrm{PGE}_{2}$ and 6-keto-PGF1 $\alpha$, a stable metabolite of prostacyclin, were measured using a $\mathrm{PGE}_{2}$ parameter assay kit. $\mathrm{PGE}_{2}$ release was significantly decreased in the $10 \mu \mathrm{M}$ treatment group (Fig. 5).

The expression levels of of HPGD following HCQ stimulation in RA-FLS were determined by western blotting. Following stimulation with HCQ $(10 \mu \mathrm{M})$, the cells were collected at different time points $(0.5,1,3,6,12$ and $24 \mathrm{~h})$. The protein expression levels of HPGD increased in a time-dependent manner (Fig. 6). To investigate whether treatment with HCQ may affect the signal transduction pathway, the $\mathrm{PGE}_{2}$ metabolic enzymes were assessed by western blotting. The cells were incubated with HCQ for $24 \mathrm{~h}$ in the presence of several signal transduction pathway inhibitors, including Wortmannin (PI3K inhibitor), SB203580 (p38 MAP kinase inhibitor) and PD98059 (ERK inhibitor). These inhibitors were added to the RA-FLS $0.5 \mathrm{~h}$ prior to HCQ stimulation. It was demonstrated that the expression of HPGD was suppressed by wortmannin and PD98059 (Fig. 7A and B). However, SB203580 revealed no effect on the expression of HPGD (Fig 7B). The expression levels of COX1, COX2 and mPGES1 were assessed. The cells were incubated with HCQ for $24 \mathrm{~h}$ in the presence of several signal pathway inhibitors. All the inhibitors suppressed the expression levels of COX2 induced by HCQ. The expression 

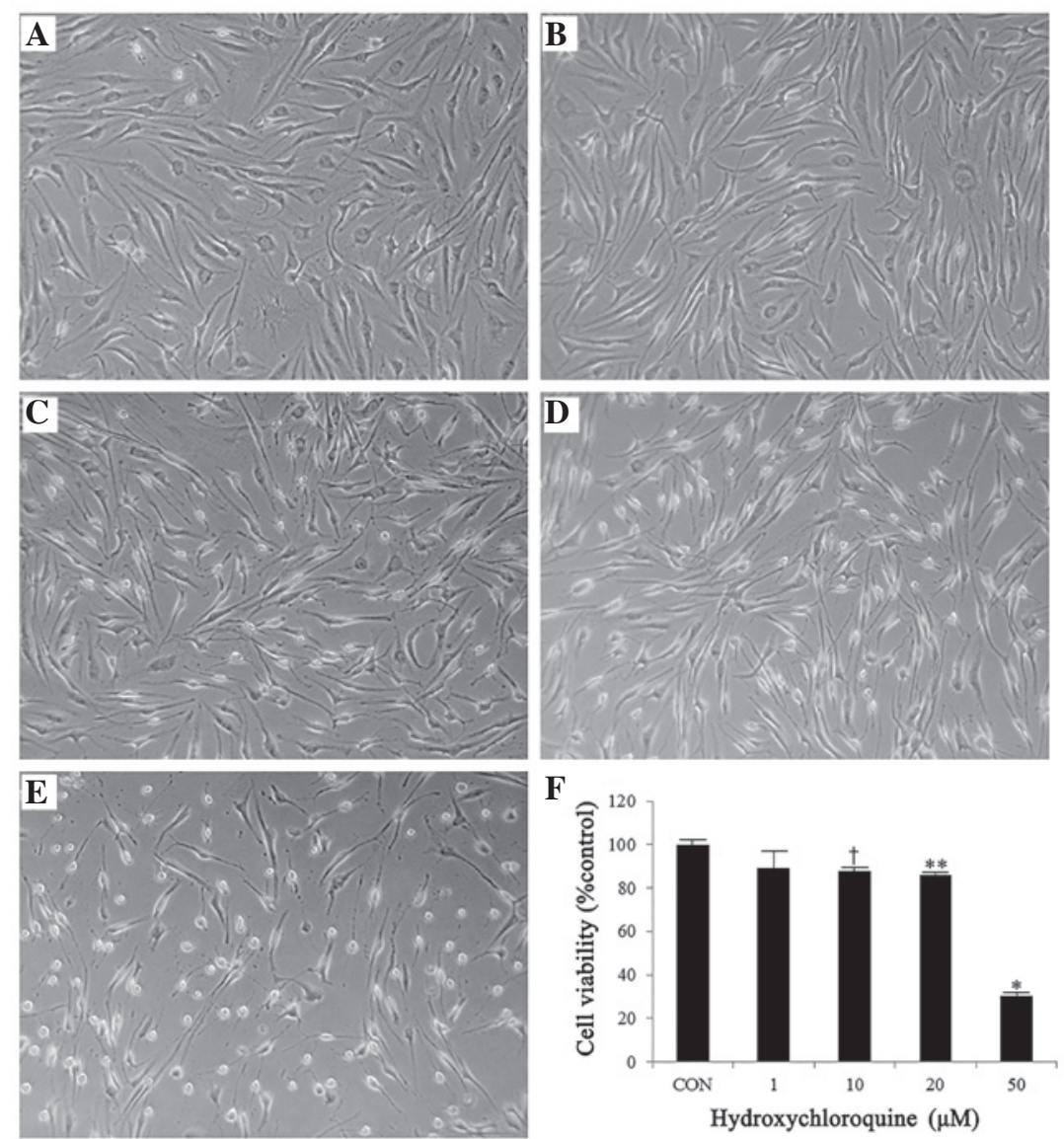

Figure 4. Effects of hydroxychloroquine on the viability of RA-FLS. The RA-FLS were serum-starved for $2 \mathrm{~h}$ and subsequently treated with different concentrations of hydroxychloroquine for $24 \mathrm{~h}$. The viability of the cells was determined using an MTS assay kit. The data are shown as the mean \pm standard deviation. (A) CON, (B) 1, (C) 10, (D) 20 or (E) $50 \mu \mathrm{M}$ and (F) graphs demonstrated the cell viability $\left(38.51 \pm 2.97,{ }^{*} \mathrm{P}<0.001 ; 93.38 \pm 2.83,{ }^{* *} \mathrm{P}=0.012 ; 94.78 \pm 3.31\right.$, ${ }^{\dagger} \mathrm{P}=0.037$, compared with the CON). CON, control; RA-FLS, rheumatoid arthritis fibroblast-like synoviocytes.

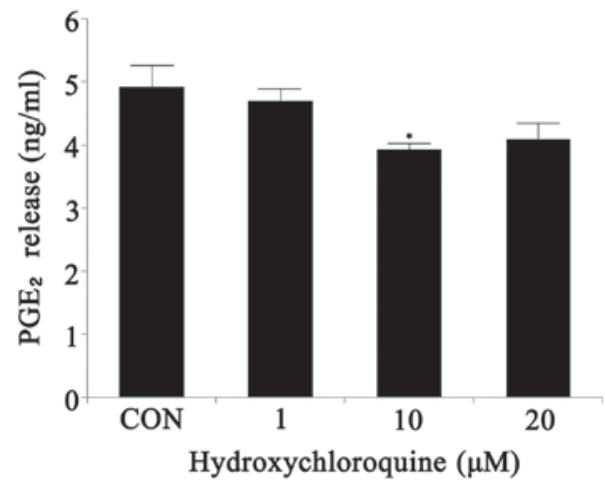

Figure 5. Effects of hydroxychloroquine on the release of PGE2. The RA-FLS were serum-starved for $2 \mathrm{~h}$ and subsequently treated with different concentrations of hydroxychloroquine for $24 \mathrm{~h}$. The quantities of PGE2 and 6-keto-PGF1 $\alpha$ were measured using a PGE2 parameter assay kit. The data are shown as the mean \pm standard deviation $\left(3.92 \pm 0.09,{ }^{*} \mathrm{P}=0.045\right.$, compared with the CON). PGE2, prostaglandin E2; CON, control; RA-FLS, rheumatoid arthritis fibroblast-like synoviocytes.

levels of COX1 and mPGES1 were not affected by treatment with HCQ and the inhibitors (Fig. 7C and D). The level of PGE $\mathrm{P}_{2}$ following stimulation of HCQ with inhibitors was detected by ELISA (Fig. 8). Wortmannin, PD98059 and SB203580 were added $0.5 \mathrm{~h}$ prior to HCQ stimulation. The decrease of HCQ-induced $\mathrm{PGE}_{2}$ release, was reversed by treatment with
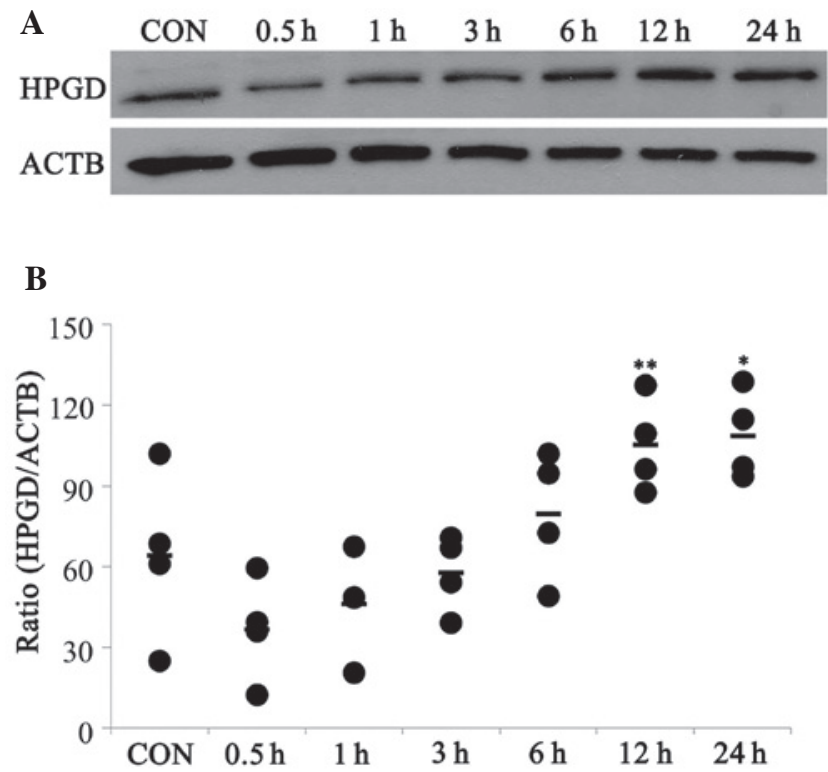

Figure 6. Expression of HPGD following hydroxychloroquine stimulation. The RA-FLS were serum-starved for $2 \mathrm{~h}$ and subsequently treated with hydroxychloroquine $(10 \mu \mathrm{M})$ for $0.5,1,3,6,12$ and $24 \mathrm{~h}$. (A) The expression levels of HPGD in RA-FLS was determined by immunoblotting. ACTB was used as an internal control. (B) The immunoblotting was quantified and the data are expressed as the mean \pm standard deviation $\left(111.23 \pm 16.07,{ }^{*} \mathrm{P}=0.012\right.$; $106.99 \pm 16.82,{ }^{* *} \mathrm{P}=0.019$, compared with the $\left.\mathrm{CON} ; \mathrm{n}=4\right)$. CON, control; HPGD, 15-hydroxyprostaglandin dehydrogenase; ACTB, $\beta$-actin; RA-FLS, rheumatoid arthritis fibroblast-like synoviocytes. 
A

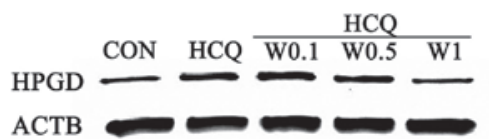

B

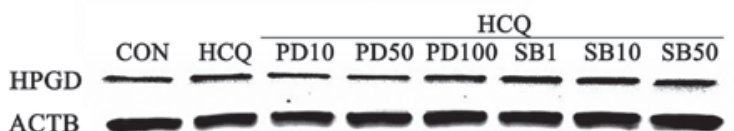

C

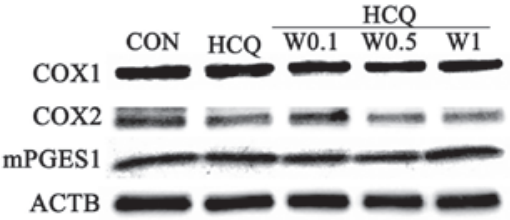

D

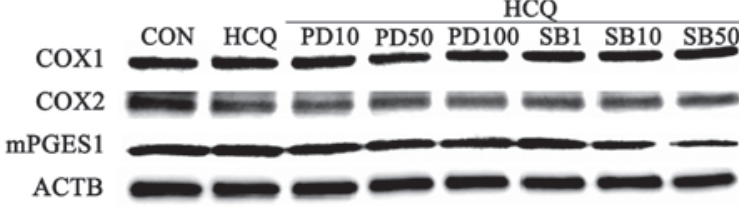

Figure 7. Expression levels of HPGD, COX and mPGES1 following HCQ stimulation by wortmannin, PD98059 and SB203580. The RA-FLS were serum-starved for $2 \mathrm{~h}$ and subsequently treated with HCQ $(10 \mu \mathrm{M})$ for $24 \mathrm{~h}$. Wortmannin (0.1,0.5 and $1 \mathrm{nM})$, PD98059 $(10,50$ and $100 \mu \mathrm{M})$ and SB203580 $(1,10$ and $50 \mu \mathrm{M})$ were added $0.5 \mathrm{~h}$ prior to HCQ stimulation. The expression levels of (A and B) HPGD and (C and D) COX1, COX2 and mPGES1 in RA-FLS following the different treatments. RA-FLS, rheumatoid arthritis fibroblast-like synoviocytes; CON, control; HCQ, hydroxychloroquine; W, wortmannin; PD, PD98059; SB, SB203580; HPGD, 15-hydroxyprostaglandin dehydrogenase; ACTB, $\beta$-actin; COX, cyclooxygenase; mPGES1, microsomal prostaglandin E2 synthase 1.

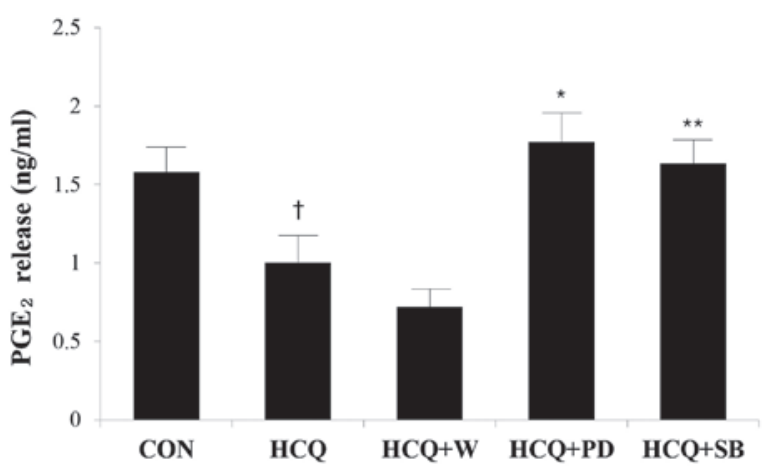

Figure 8. Levels of PGE2 release upon hydroxychloroquine stimulation by wortmannin, PD98059 and SB203580. The quantities of PGE2 and 6-keto-PGF1 $\alpha$ were measured by enzyme immunoassay. Wortmannin $(1 \mathrm{nM})$, PD98059 $(10 \mu \mathrm{M})$ and SB203580 $(50 \mu \mathrm{M})$ were added to the cells $0.5 \mathrm{~h}$ prior to HCQ $(10 \mu \mathrm{M})$ stimulation. The data are expressed as the mean \pm standard deviation $\left(1.78 \pm 0.18,{ }^{*} \mathrm{P}=0.02 ; 1.63 \pm 0.15,{ }^{* *} \mathrm{P}=0.031\right.$, compared with HCQ and $1.00 \pm 0.17,{ }^{\dagger} \mathrm{P}=0.050$, compared with the CON). PGE2, prostaglandin $\mathrm{E} 2$; CON, control; HCQ, hydroxychloroquine; W, wortmannin; PD, PD98059; SB, SB203580.

PD98059 and SB203580 (Fig. 8). Therefore, HCQ-induced PGE $_{2}$ alterations may be associated with the MAP kinase pathway. The involvement of signal transduction pathways may be used to further assess the mechanism of stimulation of the expression of HPGD by HCQ. The expression levels of SAPK/JNK, ERK and $\mathrm{p} 38$ following treatment with HCQ and inhibitor-treated RA-FLS were investigated. SAPK/JNK was phosphorylated

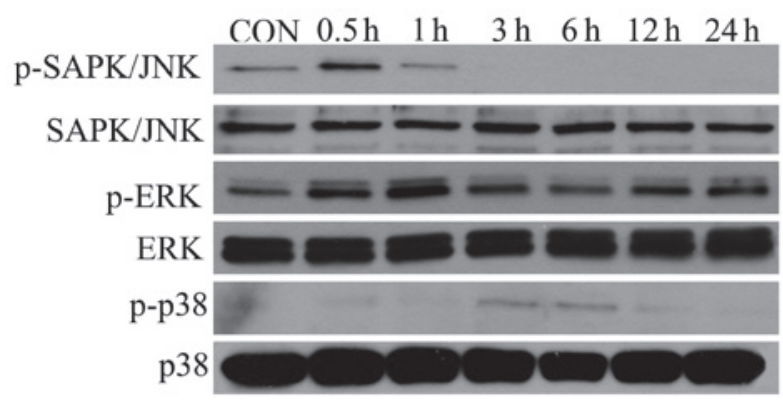

Figure 9. Phosphorylation of SAPK/JNK, ERK and p38 following hydroxychloroquine stimulation. The RA-FLS were serum-starved for $2 \mathrm{~h}$ and subsequently treated with hydroxychloroquine $(10 \mu \mathrm{M})$ for $0.5,1,3,6,12$ and 24 h. CON, control; RA-FLS, rheumatoid arthritis fibroblast-like synoviocytes; p, phosphorylated.

$0.5 \mathrm{~h}$ following HCQ stimulation $(10 \mu \mathrm{M})$ and then returned to background levels at $1 \mathrm{~h}$. ERK was phosphorylated $1 \mathrm{~h}$ following HCQ stimulation and then returned to background levels at $3 \mathrm{~h}$. p38 was phosphorylated $3 \mathrm{~h}$ following HCQ stimulation and the phosphorylation levels were maintained until $6 \mathrm{~h}$ and then returned to background levels at $12 \mathrm{~h}$ (Fig. 9).

\section{Discussion}

The association between $\mathrm{PGE}_{2}$ metabolism and the pathology of RA is well known and the majority of previous studies have focused on $\mathrm{PGE}_{2}$ and the associated enzymes. However, HPGD, the enzyme responsible for the degradation of $\mathrm{PGE}_{2}$, has received little attention in the pathology of RA, although its expression level ultimately affects the level of $\mathrm{PGE}_{2}$. HPGD is the key enzyme responsible for the metabolic inactivation of $\mathrm{PGE}_{2}$ (18). Previous studies demonstrated a $\mathrm{PGE}_{2}$ mechanism involving HPGD. COX enzymes catalyze the conversion of arachidonic acid into prostaglandin H2. mPGES1 enzymes subsequently catalyze the conversion of prostaglandin $\mathrm{H} 2$ into $\mathrm{PGE}_{2} . \mathrm{PGE}_{2}$ is degraded and inactivated by the initial oxidation of their 15(S)-hydroxyl group, and this is catalyzed by HPGD (19). Previous studies have demonstrated increased quantities of COX2 and mPGES1 in OA cartilage in response to mechanical stress (5). Decreased quantities of HPGD in RA-FLS (2), eutopic endometrium (6), colonic mucosa (20) and gastric carcinoma (21) have also been reported. The expression of HPGD was absent in colonic mucosa and gastric carcinoma (20,21). Additionally, HPGD activity was absent in lung adenocarcinoma and colon cancer cells $(22,23)$. Therefore, it is likely that the increased level of $\mathrm{PGE}_{2}$ in RA is a consequence of reduced catabolism and increased synthesis. Based on these previous findings, it was important to determine whether the expression levels of HPGD were changed in patients with RA. The present study demonstrated reduced expression levels of HPGD in the RA synovial tissue and FLS. The protein expression levels of HPGD were determined in RA-FLS treated with several DMARDs, to investigate whether the DMARDs affected the expression of HPGD in RA-FLS. The expression levels of HPGD were increased by treatment with DMARDs, including MTX, HCQ, SSZ and infliximab. Conventional DMARDs are generally offered as a first-line treatment for patients with RA. Biological DMARDs offer 
a valuable treatment alternative for patients with suboptimal response or intolerance to conventional DMARDs or when continued conventional DMARDs therapy fails (24).

HCQ, a DMARDs used in the present study, is a drug that has been used to treat autoimmune disorders, including RA and systemic lupus erythematosus (25-27). However, the specific mechanism for its pharmacological action remains to be elucidated. The present study investigated whether HCQ induced the expression of HPGD and the signal transduction pathway involved in this phenomenon. The expression levels of HPGD were increased time-dependently following treatment with HCQ (Fig. 6). Treatment with HCQ may affect the expression levels of HPGD, COX1, COX2 and mPGES1 (Fig. 7). These changes were affected by MAP-kinase inhibitors, including PD98059 and SB203580 (Fig. 7C and D). These results suggest that $\mathrm{HCQ}$ may affect the expression levels of HPGD via the MAP-kinase pathway in RA-FLS. In addition, these findings that $\mathrm{PGE}_{2}$ regulation occurs via $\mathrm{COX} 2$ corroborate a previous observation that HCQ negatively affected the activity of COX2 (28). Previous studies have reported that inhibitors of ERK and p38 inhibited the upregulation of COX2 in human follicular dendritic cells $(29,30)$. There are increased quantities of COX 2 and mPGES1 in the inflamed mucosa of inflammatory bowel disease $(31,32)$. Otani et al (20) reported that the reduced expression levels of HPGD contributes to the increased levels of $\mathrm{PGE}_{2}$ observed in the inflamed mucosa of patients with inflammatory bowel disease. IL-4 upregulates the levels of HPGD by increasing gene transcription and decreasing protein turnover, and the upregulation can be mediated by JAK-STAT6, MAP kinases, PI3K/Akt and PKC pathways (33). The present study also demonstrated that HCQ inhibited COX2 and induced HPGD via the MAP-kinase pathway.

In conclusion, HPGD is weakly expressed in synovial tissue in conditions associated with inflammatory responses, including OA and RA. The expression levels of HPGD were lower in RA tissue compared with normal tissues. Treatment with HCQ affected the pathology of RA through the increased expression levels of HPGD and decreased levels of $\mathrm{PGE}_{2}$, and this may be associated with the MAP-kinase pathway. The exact role of HPGD as a potential target for the treatment of RA remains to be elucidated.

\section{Acknowledgements}

This study was supported by the Soonchunhyang University Research Fund and the Basic Science Research Program of the National Research Foundation of Korea, funded by the Ministry of Education, Science, and Technology (no. 2010-0024573).

\section{References}

1. Lee DM and Weinblatt ME: Rheumatoid arthritis. Lancet 358 903-911, 2001

2. Gheorghe KR, Sadique S, Leclerc P, et al: Limited effect of anti-rheumatic treatment on 15-prostaglandin dehydrogenase in rheumatoid arthritis synovial tissue. Arthritis Res Ther 14: R121, 2012.

3. Westman M, Korotkova M, afKlint E, et al: Expression of microsomal prostaglandin Esynthase 1 in rheumatoid arthritis synovium. Arthritis Rheum 50: 1774-1780, 2004.

4. Tai HH: Prostaglandin catabolic enzymes as tumor suppressors. Cancer Metastasis Rev 30: 409-417, 2011.
5. Gosset M, Berenbaum F, Levy A, Pigenet A, Thirion S, Cavadias $S$ and Jacques C: Mechanical stress and prostaglandin E2 synthesis in cartilage. Biorheology 45: 301-320, 2008

6. Lousse JC, Defrère S, Colette S, Van Langendonckt A and Donnez J: Expressionof eicosanoid biosynthetic and catabolic enzymes in peritoneal endometriosis. Hum Reprod 25: 734-741, 2010.

7. Celis JE, Ostergaard M, Basse B, et al: Loss of adipocyte-type fatty acid binding protein and other protein biomarkers is associated with progression of human bladder transitional cell carcinomas. Cancer Res 56: 4782-4790, 1996.

8. Gee JR, Montoya RG, Khaled HM, Sabichi AL and Grossman HB: Cytokeratin 20, AN43, PGDH and COX-2 expression in transitional and squamous cell carcinoma of the bladder. Urol Oncol 21: 266-270, 2003.

9. Tseng-Rogenski S, Gee J, Ignatoski KW, et al: Loss of 15-hydroxyprostaglandin dehydrogenase expression contributes to bladder cancer progression. Am J Pathol 176: 1462-1468, 2010.

10. Yan M,Rerko RM,Platzer P, et al: 15-Hydroxyprostaglandin dehydrogenase, a COX-2 oncogeneantagonist, is a TGF-beta-induced suppressor of human gastrointestinal cancers. Proc Natl Acad Sci USA 101: 17468-17473, 2004

11. Gosset M, Berenbaum F, Salvat C, et al: Crucial role of visfatin/pre-B cell colony-enhancing factor in matrix degradation and prostaglandin E2 synthesis in chondrocytes: Possible influence on osteoarthritis. Arthritis Rheum $58: 1399-1409,2008$.

12. Smolen JS, van der Heijde D, Machold KP, Aletaha D and Landewé R: Proposal for a new nomenclature of disease-modifying antirheumatic drugs. Ann Rheum Dis 73: 3-5, 2014.

13. Mercer E, Rekedal L, Garg R, Lu B, Massarotti EM and Solomon DH: Hydroxychloroquine improves insulin sensitivity in obese non-diabeticindividuals. Arthritis Res Ther 14: R135, 2012.

14. Unübol M, Ayhan M and Guney E: Hypoglycemia induced by hydroxychloroquine in a patient treated for rheumatoid arthritis. J Clin Rheumatol 17: 46-47, 2011.

15. Nah SS, Won HJ, Park HJ, et al: Melatonin inhibits human fibroblast-like synoviocyte proliferation via extra cell ular signal-regulated protein kinase/P21(CIP1)/P27(KIP1) pathways. J Pineal Res 47: 70-74, 2009.

16. Clive R, Shi SR, Chen C, et al: Comparative study of antigen retrieval heating methods: Microwave, microwave and pressure cooker, autoclave, and steamer. Biotechnic \& Histochemistry 5: 263-270, 1996

17. Kim HJ, Eom CY, Kwon J, et al: Roles of interferon-gamma and its target genes in schizophrenia: Proteomics-based reverse genetics from mouse to human. Proteomics 12: 1815-1829, 2012.

18. Tai HH, Ensor CM, Tong M, Zhou H and Yan F: Prostaglandin catabolizing enzymes. Prostaglandins Other Lipid Mediat 68-69: 483-493, 2002

19. Tai HH, Cho H, Tong M and Ding Y: NAD+-linked 15-hydroxyprostaglandindehydrogenase: structure and biological functions. Curr Pharm Des 12: 955-962, 2006.

20. Otani T, Yamaguchi K, Scherl E, et al: Levels of $\mathrm{NAD}(+)$-dependent 15-hydroxyprostaglandin dehydrogenase are reduced in inflammatory bowel disease: evidence for involvement of TNF-alpha. Am J Physiol Gastrointest Liver Physiol 290: G361-G368, 2006.

21. Lou LH, Jing DD, Lai YX, Lu YY, Li JK and Wu K: 15-PGDH is reduced and induces apoptosis and cell cycle arrest in gastric carcinoma. World J Gastroenterol 18: 1028-1037, 2012.

22. Tong $\mathrm{M}$ and Tai HH: 15-Hydroxyprostaglandin dehydrogenase can be induced by dexamethasone and other glucocorticoids at the therapeutic level inA549 human lung adenocarcinoma cells. Arch Biochem Biophys 435: 50-55, 2005.

23. Chi X, Freeman BM, Tong $M$, Zhao Y and Tai HH: 15 -Hydroxy prostaglandindehydrogenase (15-PGDH) is up-regulated by flurbiprofen and other non-steroidal anti-inflammatory drugs in human colon cancer HT29 cells. Arch Biochem Biophys 487: 139-145, 2009.

24. Thwaites $\mathrm{C}$ and Finney A: Rheumatoid arthritis. 2: Exploring treatment options to achieve early control and remission. Nurs Times 106: 18-20, 2010.

25. Min JK, Lee HJ, Lee WS, et al: Effects of hydroxychloroquine on the metabolism of fas ligand of T cells. J Korean Rheum Assoc 7: 127-139, 2000.

26. Mackenzie AH: Pharmacologic actions of 4-aminoquinoline compounds. Am J Med 75: 5-10, 1983.

27. Dubois EL: Antimalarials in the management of discoid and systemic lupus erythematosus. Semin Arthritis Rheum 8: 33-51, 1978. 
28. Ben-Chetrit E, Fischel R, Hinz B and Levy M: The effects of colchicine and hydroxychloroquine on the cyclo-oxygenases COX-1 and COX-2. Rheumatol Int 25: 332-335, 2005.

29. Cho W, Kim Y, Jeoung DI, Kim YM and Choe J: IL-4 and IL-13 suppress prostaglandins production in human follicular dendritic cells by repressingCOX-2 and mPGES-1 expression through JAK1 and STAT6. Mol Immunol 48: 966-972, 2011.

30. Nagano S, Otsuka T, Niiro H, et al: Molecular mechanisms of lipopolysaccharide-induced cyclooxygenase- 2 expression inhuman neutrophils: involvement of the mitogen-activated protein kinasepathway and regulation by anti-inflammatory cytokines. Int Immunol 14: 733-740, 2002.
31. Singer II, Kawka DW, Schloemann S, Tessner T, Riehl T and Stenson WF: Cyclooxygenase 2 is induced in colonic epithelial cells in inflammatory bowel disease. Gastroenterology 115: 297-306, 1998.

32. Subbaramaiah K, Yoshimatsu K, Scherl E, et al: Microsomal prostaglandin E synthase-1 is overexpressed in inflammatory bowel disease. Evidence for involvement of the transcription factor Egr-1. J Biol Chem 279: 12647-12658, 2004.

33. Chi $\mathrm{X}$ and Tai HH: Interleukin-4 up-regulates 15-hydroxyprostaglandindehydrogenase (15-PGDH) in human lung cancer cells. Exp Cell Res 316: 2251-2259, 2010. 